\title{
The Effects of Non-Insulin-dependent Diabetes Mellitus on the Kinetics of Onset of Insulin Action in Hepatic and Extrahepatic Tissues
}

\author{
D. Turk, A. Alzaid, S. Dinneen, K. S. Nair, and R. Rizza \\ Endocrine Research Unit, Department of Medicine, Mayo Clinic, Rochester, Minnesota 55905
}

\begin{abstract}
The mechanism(s) of insulin resistance in non-insulin-dependent diabetes mellitus remains ill defined. The current studies sought to determine whether non-insulin-dependent diabetes mellitus is associated with $(a)$ a delay in the rate of onset of insulin action, $(b)$ impaired hepatic and extrahepatic kinetic responses to insulin, and $(c)$ an alteration in the contribution of gluconeogenesis to hepatic glucose release. To answer these questions, glucose disappearance, glucose release, and the rate of incorporation of ${ }^{14} \mathrm{CO}_{2}$ into glucose were measured during 0.5 and $1.0 \mathrm{mU} / \mathrm{kg}^{-1}$ per min $^{-1}$ insulin infusions while glucose was clamped at $\sim 95$ $\mathrm{mg} / \mathrm{dl}$ in diabetic and nondiabetic subjects. The absolute rate of disappearance was lower $(P<0.05)$ and the rate of increase slower $(P<0.05)$ in diabetic than nondiabetic subjects during both insulin infusions. In contrast, the rate of suppression of glucose release in response to a change in insulin did not differ in the diabetic and nondiabetic subjects during either the low (slope 30-240 min:0.02 \pm 0.01 vs $0.02 \pm 0.01)$ or high $(0.02 \pm 0.00$ vs $0.02 \pm 0.00)$ insulin infusions. However, the hepatic response to insulin was not entirely normal in the diabetic subjects. Both glucose release and the proportion of systemic glucose being derived from ${ }^{14} \mathrm{CO}_{2}$ (an index of gluconeogenesis) was inappropriately high for the prevailing insulin concentration in the diabetic subjects. Thus non-insulin-dependent diabetes mellitus slows the rate-limiting step in insulin action in muscle but not liver and alters the relative contribution of gluconeogenesis and glycogenolysis to hepatic glucose release. (J. Clin. Invest. 1995. 95:755-762.) Key words: insulin resistance • gluconeogenesis • glucose turnover $\bullet$ rate of onset of insulin action
\end{abstract}

\section{Introduction}

Non-insulin-dependent diabetes mellitus (NIDDM) ${ }^{1}$ is associated with both hepatic and extrahepatic insulin resistance (1-

This work was presented in part at Southern Section American Federation for Clinical Research, New Orleans, LA, 3-5 February 1994.

Address correspondence to Robert A. Rizza, Endocrine Research Unit, 5-164 West Joseph, Mayo Clinic, Rochester, MN 55905. PHONE: (507) 255-6515; FAX: (507) 255-4828.

Received for publication 30 March 1994 and in revised form 3 August 1994.

1. Abbreviation used in this paper: NIDDM, non-insulin-dependent diabetes mellitus.

\section{J. Clin. Invest.}

(C) The American Society for Clinical Investigation, Inc.

0021-9738/95/02/0755/08 $\$ 2.00$

Volume 95, February 1995, 755-762
5). The hyperinsulinemic euglycemic clamp technique has been extensively used to establish that hepatic glucose release is inappropriately increased and glucose uptake inappropriately decreased relative to the prevailing insulin concentration in NIDDM (1-5). However, these conclusions are based on steady state measurements performed after several hours of insulin infusion. In nondiabetic humans, the rapid increase in insulin concentration that occurs after food ingestion results in prompt suppression of hepatic glucose release and stimulation of glucose uptake (6-9). A delay in insulin secretion causes postprandial hyperglycemia (10). Therefore the rate of onset of insulin action is likely to be as, if not more, important than the absolute effect eventually achieved at any given insulin concentration.

The mechanism of insulin resistance in hepatic and extrahepatic tissues in people with NIDDM remains ill defined. Previous studies have suggested that the rate of insulin-induced stimulation of muscle glucose uptake is slower in diabetic than nondiabetic individuals (11). It is currently not known whether the rate of suppression of hepatic glucose release by insulin also is slower. A similar decrease in the rate of response of both liver and muscle to insulin would support a common defect in insulin action. In contrast, a delay in stimulation of glucose uptake but a normal rate of suppression of hepatic glucose release would indicate that insulin resistance has slowed the rate-limiting step in insulin action in muscle but not the liver. Even then the hepatic response to insulin may not be completely normal if the relative contribution of gluconeogenesis and glycogenolysis to hepatic glucose output differs from that observed in nondiabetic humans. Several recent studies have reported that gluconeogenesis is increased in the postabsorptive state in NIDDM $(12,13)$. There is limited information as to whether or not gluconeogenesis also is resistant to insulin.

The current studies therefore were undertaken to test the hypothesis that at insulin concentrations spanning the physiologic range, the rate of onset of insulin action is slower in both hepatic and extrahepatic tissues in diabetic than nondiabetic humans. We also sought to test the hypothesis that insulininduced suppression of gluconeogenesis is impaired in NIDDM, thereby causing newly synthesized glucose to make a proportionately greater contribution to hepatic glucose release in diabetic than nondiabetic humans.

\section{Methods}

Subjects. After approval from the Mayo Institutional Review Board, seven volunteers with NIDDM and seven matched nondiabetic volunteers (Table I) gave informed written consent to participate in the study. The glycosylated hemoglobin concentrations in the diabetic volunteers averaged $9.0 \pm 1.0 \%$ (normal range $4-7 \%$ ) at the time of entry into the study. Two of the seven diabetic subjects were being treated with diet alone and the remaining five with diet plus a sulfonylurea agent. In the latter individuals, the sulfonylurea was discontinued two weeks before 
Table I. Subject Characteristics

\begin{tabular}{lcc}
\hline & Diabetic & Nondiabetic \\
\hline Age (yr) & $53 \pm 4$ & $51 \pm 3$ \\
Gender (male/female) & $5 / 2$ & $4 / 3$ \\
Body mass index $\left(\mathrm{kg} / \mathrm{m}^{2}\right)$ & $28 \pm 2$ & $26 \pm 1$ \\
Waist/hip ratio & $0.91 \pm 0.03$ & $0.91 \pm 0.03$ \\
\hline
\end{tabular}

study. The nondiabetic subjects had normal fasting blood glucose concentrations and no first degree relatives with diabetes mellitus.

Experimental design. All studies were conducted in the Mayo General Clinical Research Center. Subjects were studied on two occasions separated by at least $1 \mathrm{wk}$. The volunteers were admitted to the Mayo General Clinical Research Center the night before the study and received a standard mixed meal ( 636 calories, $48 \%$ carbohydrate, $32 \%$ fat, and $19 \%$ protein) at 1800-1830. After placement of an 18-gauge catheter in a forearm vein, an intravenous infusion of either insulin (diabetic volunteers) or saline (nondiabetic volunteers) was initiated. The insulin infusion rate was varied according to the algorithm of White et al. (14) so as to achieve and maintain euglycemia during the night. At 0630 the next morning, an additional 18-gauge catheter was placed in an antecubital vein for infusion of glucose, insulin, potassium chloride, and tracers. A needle also was placed retrogradely into a dorsal vein of the opposite hand. This hand was then placed into a heated unit to provide arterialized venous blood samples.

Primed-continuous infusions of $\left[6-{ }^{3} \mathrm{H}\right]$ glucose $(8.0 \mu \mathrm{Ci}, 0.08 \mu \mathrm{Ci} /$ $\min$ ) and $\mathrm{H}^{14} \mathrm{CO}_{3}(110 \mu \mathrm{Ci}, 1.1 \mu \mathrm{Ci} / \mathrm{min}$ ) (both from New England Nuclear, Boston, MA) were started at $0700 .\left[{ }^{13} \mathrm{C}\right]$ leucine was also infused as part of a separate protocol. The $\left[6-{ }^{3} \mathrm{H}\right]$ glucose was confirmed to be $>99 \%$ pure by HPLC. The nocturnal insulin (in the diabetic subjects) or saline (in the nondiabetic subjects) was discontinued at 1100 and an unprimed, continuous infusion of insulin (Humulin R; Eli Lilly and Co., Indianapolis, IN) started at a rate of either 0.5 or 1.0 $\mathrm{mU} / \mathrm{kg}^{-1}$ per $\mathrm{min}^{-1}$. These insulin infusions were stopped at 1500 . The insulin was mixed with $0.9 \%$ normal saline and $0.1 \%$ human serum albumin (Miles Inc., Elkhart, IN). Glucose containing $\left[6-{ }^{3} \mathrm{H}\right]$ glucose was infused in amounts sufficient to maintain euglycemia (15).

Analytical techniques. Blood and expired air were collected at regular intervals as previously described (16). Plasma glucose concentration was measured immediately upon collection using a glucose oxidase method (glucose and lactate analyzer; Yellow Springs Instrument Co., Yellow Springs, $\mathrm{OH}$ ). Additional blood was placed on ice, centrifuged at $4^{\circ} \mathrm{C}$, separated, and stored at $-20^{\circ} \mathrm{C}$ until assay. Plasma insulin, cpeptide, glucagon, growth hormone concentrations, and glucose and breath $\left({ }^{14} \mathrm{CO}_{2}\right)$ specific activities were determined as previously described $(7,15-17)$. Lactate concentrations were measured using a lactate oxidase method (glucose and lactate analyzer; Yellow Springs Instrument Co.). Plasma glycerol concentrations were measured using a peroxidase-coupled colormetric assay (18). Plasma amino acid concentrations were measured using reverse phase HPLC (19).

Calculations. Assuming as reported by Adkins-Marshall et al. (20), that the portal vein accounts for $\sim 80 \%$ of hepatic blood flow, hepatic sinusoidal insulin concentrations were calculated as $\mathrm{HSI}=\left[\left(\mathrm{PI}_{\mathrm{t}} \times 4\right)\right.$ $\left.+\left(\mathrm{AI}_{t}\right)\right] / 5$ where $\mathrm{PI}_{t}$ and $\mathrm{AI}_{t}$ are portal and arterialized venous insulin concentrations present at time $t$. Portal insulin concentrations were calculated as suggested by Hother-Nielsen et al. (21) assuming that the portal venous/peripheral venous gradient is $2.4 / 1$ in the fasting state. Therefore $\mathrm{PI}_{0}$ was calculated as $2.4 \times \mathrm{AI}_{0}$ and $\mathrm{PI}_{\mathrm{t}}=\mathrm{AI}_{\mathbf{t}}+\left[\left(\mathrm{PI}_{0}-\mathrm{AI}_{0}\right)\right.$ $\left.\times\left(\mathrm{CP}_{t} / \mathrm{CP}_{0}\right)\right]$ where $\mathrm{CP}$ is arterialized venous c-peptide concentrations and the subscript 0 refers to the mean basal concentration present before the low and high dose insulin infusions (i.e., from $-30-0 \mathrm{~min}$ ). Since the diabetic subjects were being infused with insulin during the basal period, $\mathrm{PI}_{0}$ and $\mathrm{CP}_{0}$ were set equal to the mean values present in the nondiabetic subjects. This assumes that insulin and c-peptide clearance in the fasting state is the same in diabetic and nondiabetic individuals.

Glucose appearance and disappearance were calculated in each subject using Steele's non-steady state equations (22) as modified by DeBodo et al. (23). The glucose volume of distribution was assumed to equal $200 \mathrm{ml} / \mathrm{kg}$ and the pool correction factor 0.65 . Glucose specific activity was smoothed using the OOPSEG smoothing program (24) provided courtesy of Drs. Bradley and Bergman. Hepatic glucose release was calculated by subtracting the glucose infusion rate from the tracer determined rate of appearance. The percentage of glucose derived from bicarbonate was estimated by dividing the plasma $\left[6-{ }^{14} \mathrm{C}\right]$ glucose specific activity by the ${ }^{14} \mathrm{CO}_{2}$ breath specific activity as previously described $(16,17)$. As with all current isotopic dilution approaches, this method is influenced by dilution of the tracer within the oxaloacetate pool (25, 26). However, changes in intramitochondrial dilution have been shown to be small under a variety of circumstances (26) and if anything presumably would be greater in the diabetic subjects due to increased rates of fat oxidation (3); this would lead to an underestimation rather than an overestimation of the rate of incorporation of labeled carbon dioxide into glucose. While this approach does not measure gluconeogenesis from glycerol, contribution of glycerol to hepatic glucose release is small particularly during insulin infusion (27).

Statistical analysis. Data in the figures and text are expressed as mean \pm SEM. Basal concentrations and rates were calculated by meaning the values present during the $30 \mathrm{~min}$ before the low and high dose insulin infusions. Area below basal was calculated using the trapezoidal rule. The slope of the rate of rise in glucose disappearance and the rate of fall of hepatic glucose release was determined in each individual using linear regression analysis. The relative rates of suppression of hepatic glucose release and rate of incorporation of ${ }^{14} \mathrm{CO}_{2}$ into glucose in each individual was determined by first setting the basal value of each parameter equal to $100 \%$ then expressing the rate decline of each parameter over time as a percentage of the basal value. Results between and within groups were compared using Student's nonpaired paired and paired two-tailed $t$ tests, respectively. A $P$ value of $<0.05$ was considered to be statistically significant.

\section{Results}

Plasma glucose, c-peptide, and insulin concentrations. Fasting plasma glucose concentrations were slightly but significantly $(P<0.01)$ higher in the diabetic than nondiabetic subjects before the $0.5 \mathrm{mU} / \mathrm{kg}^{-1}$ per min ${ }^{-1}$ insulin infusion but did not differ before the $1.0 \mathrm{mU} / \mathrm{kg}^{-1}$ per $\mathrm{min}^{-1}$ insulin infusion (Fig. 1 , top panel). Plasma glucose concentrations were the same in the diabetic and nondiabetic subjects during both the low and high insulin infusions.

The overnight insulin infusion resulted in fasting peripheral insulin concentrations that were higher $(P<0.01)$ in diabetic than nondiabetic subjects before both the low $(132 \pm 25$ vs. $34 \pm 4 \mathrm{pmol} / \mathrm{liter})$ and high ( $120 \pm 21 \mathrm{vs.} 38 \pm 4$ pmol/liter $)$ insulin infusions (Fig. 1, second panel). On the other hand peripheral insulin concentrations did not differ between groups during either the low or high dose insulin infusions.

C-peptide concentrations were lower $(P<0.001)$ in diabetic subjects than nondiabetic subjects both before and during the low and high dose insulin infusions (Fig. 1, third panel). However, the magnitude of the difference decreased with time during the insulin infusions since c-peptide fell in the latter but not the former.

Although the absolute levels were higher, calculated hepatic sinusoidal insulin showed the same pattern as peripheral insulin concentrations (Fig. 1, bottom panel). Calculated hepatic sinusoidal insulin concentrations were higher $(P<0.05)$ in the diabetic than nondiabetic subjects before both the low $(143 \pm 23$ 


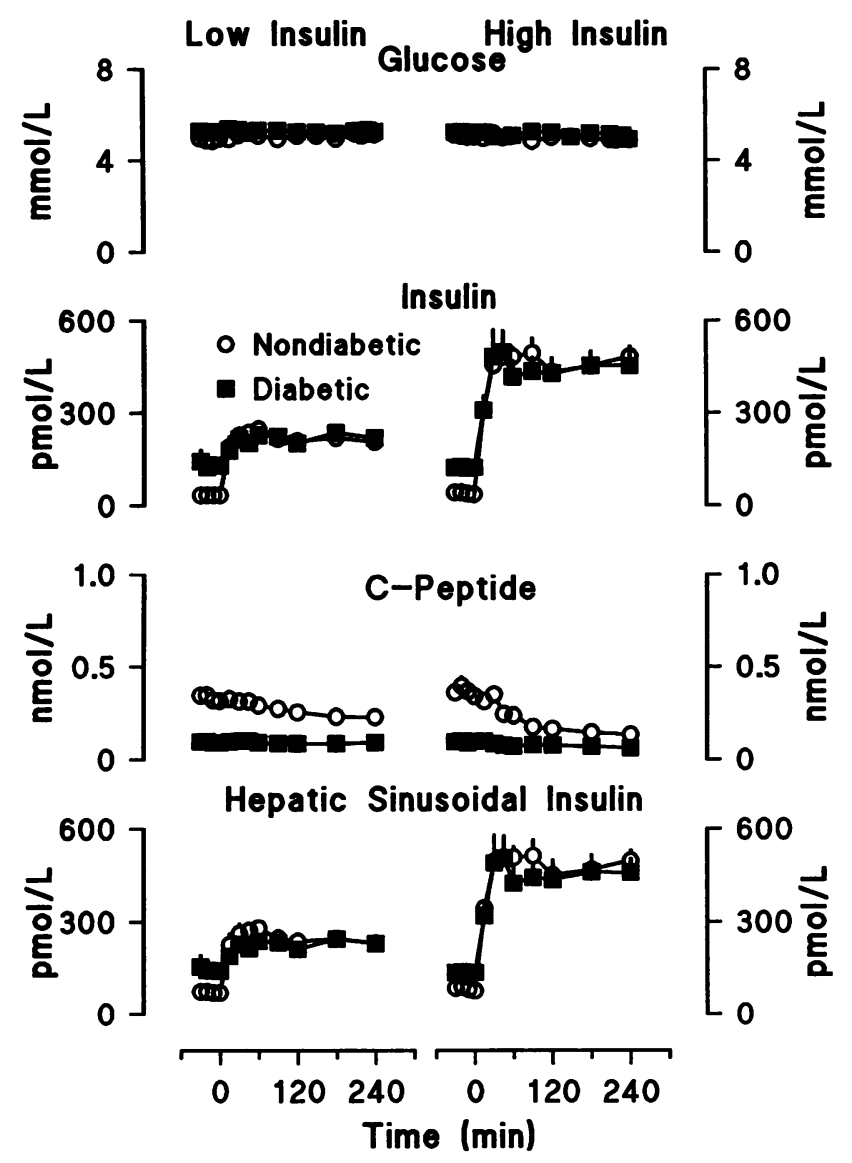

Figure 1. Glucose, insulin, c-peptide, and estimated hepatic sinusoidal insulin concentrations observed in the diabetic and nondiabetic subjects during the low (left) and high (right) dose insulin infusions. The low and high dose insulin infusions were started at time zero.

vs $71 \pm 8 \mathrm{pmol} /$ liter $)$ and high ( $131 \pm 19$ vs $82 \pm 7 \mathrm{pmol} / \mathrm{liter})$ dose insulin infusions. As with peripheral insulin concentrations, calculated hepatic sinusoidal insulin concentrations did not differ between groups during either the low or high dose insulin infusions.

Glucagon, growth hormone, and cortisol were comparable in both groups before and during both insulin infusions (data not shown).

Glucose infusion rates and glucose specific activities. The glucose infusion rate required to maintain euglycemia was lower $(P<0.01)$ in the diabetic than the nondiabetic subjects reaching rates, respectively, of $11.0 \pm 2.3$ vs $26.6 \pm 1.9 \mu \mathrm{mol} / \mathrm{kg}$ per min and $18.6 \pm 3.3$ vs $33.7 \pm 2.4 \mu \mathrm{mol} / \mathrm{kg}^{-1}$ per min ${ }^{-1}$ during the final $30 \mathrm{~min}$ of the low and high dose insulin infusions (Fig. 2 ). $\left[6-{ }^{3} \mathrm{H}\right]$ glucose specific activity increased slightly but similarly in both groups during both insulin infusions. $\left[6-{ }^{14} \mathrm{C}\right]-$ glucose specific activity fell less rapidly $(P<0.01)$ in the diabetic during both insulin infusions.

Hepatic glucose release and glucose disappearance. Hepatic glucose release was higher in the diabetic than nondiabetic subjects before both the low $\left(10.6 \pm 0.7 \mathrm{vs} 8.9 \pm 1.2 \mu \mathrm{mol} / \mathrm{kg}^{-1}\right.$ per $\left.\min ^{-1}\right)$ and high $\left(10.7 \pm 0.8\right.$ vs $7.8 \pm 0.8 \mu \mathrm{mol} / \mathrm{kg}^{-1}$ per $\left.\mathrm{min}^{-1}\right)$ dose insulin infusions, however, the difference was significant only during the latter $(P<0.05)$. The rapid increase in insulin concentrations that occurred during the $30-\mathrm{min}$ period

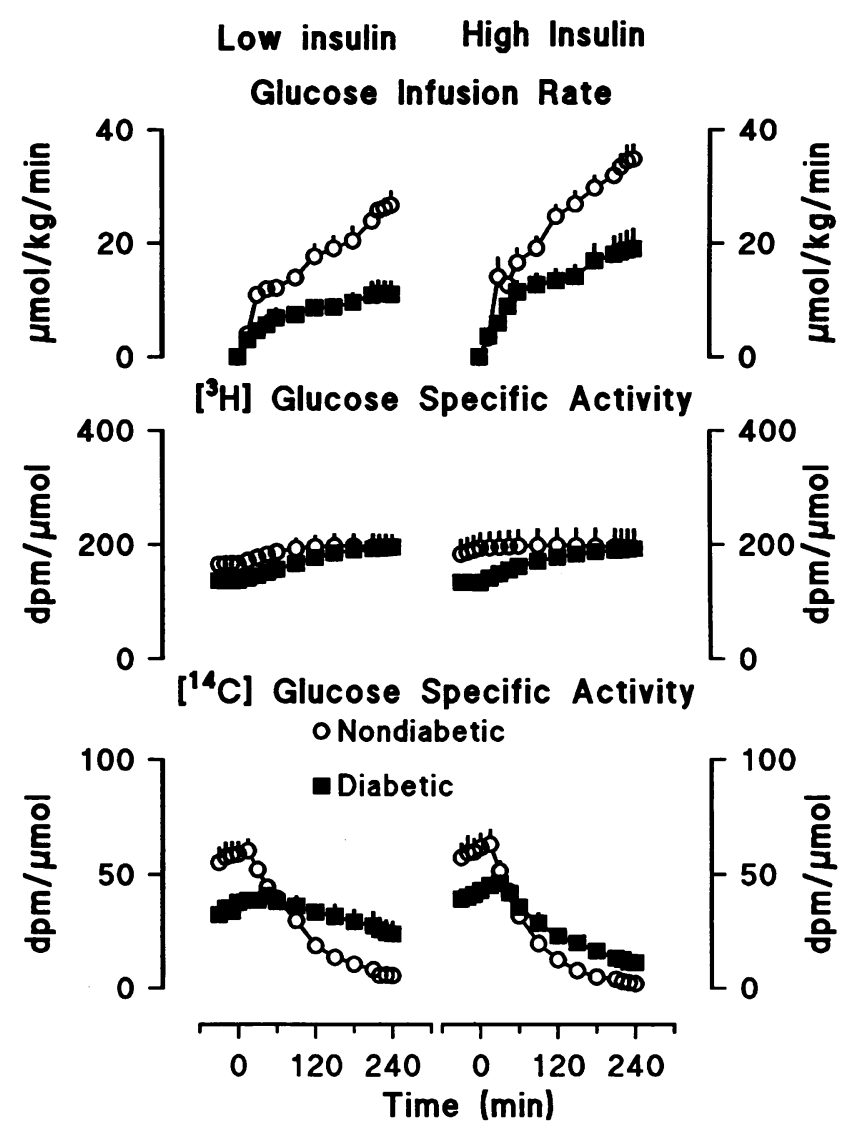

Figure 2. Glucose infusion rates required to maintain euglycemia, [6$\left.{ }^{3} \mathrm{H}\right]$ glucose specific activity, and $\left[{ }^{14} \mathrm{C}\right]$ glucose specific activity observed in the diabetic and nondiabetic subjects during the low (left) and high (right) dose insulin infusions. The low and high dose insulin infusions were started at time zero.

following initiation of the low and high dose insulin infusion resulted in a prompt decrease in hepatic glucose release in both groups (Fig. 3, bottom panels). From $30 \mathrm{~min}$ onward when insulin concentrations were equal and constant in both groups, the rate of suppression of hepatic glucose release did not differ $(P>0.75)$ in the diabetic and nondiabetic subjects during either the low (slope $30-240 \mathrm{~min}: 0.02 \pm 0.01$ vs $0.02 \pm .01 \mu \mathrm{mol} /$ $\mathrm{kg}^{-1}$ per $\mathrm{min}^{-2}$ ) or high (slope $30-240 \mathrm{~min}: 0.02 \pm 0.00 \mathrm{vs}$ $0.02 \pm 0.01 \mu \mathrm{mol} / \mathrm{kg}^{-1}$ per $\mathrm{min}^{-2}$ ) dose insulin infusions (Fig. 4). However, despite a comparable rate of fall and comparable degree of suppression below basal, hepatic glucose release during the final $30 \mathrm{~min}$ of the low dose insulin infusion remained greater $(P<0.05)$ in the diabetic than nondiabetic subjects $\left(4.3 \pm 0.8\right.$ vs $1.4 \pm 0.8 \mu \mathrm{mol} / \mathrm{kg}^{-1}$ per $\left.\mathrm{min}^{-1}\right)$. Hepatic glucose release over the same interval was slightly but not significantly greater in the diabetic subjects during the high dose insulin infusion.

Glucose disappearance increased $(P<0.01)$ within $15 \mathrm{~min}$ of initiation of the low and high dose insulin infusions in the nondiabetic (i.e., fourth circles in top panels of Fig. 3) but not the diabetic subjects (i.e., fourth squares in top panels of Fig. 3 ). Furthermore, in contrast to what was observed with glucose appearance, the rate of increase of glucose disappearance was slower from 30 min onward in diabetic subjects compared to nondiabetic subjects during both insulin infusions. Insulin had 


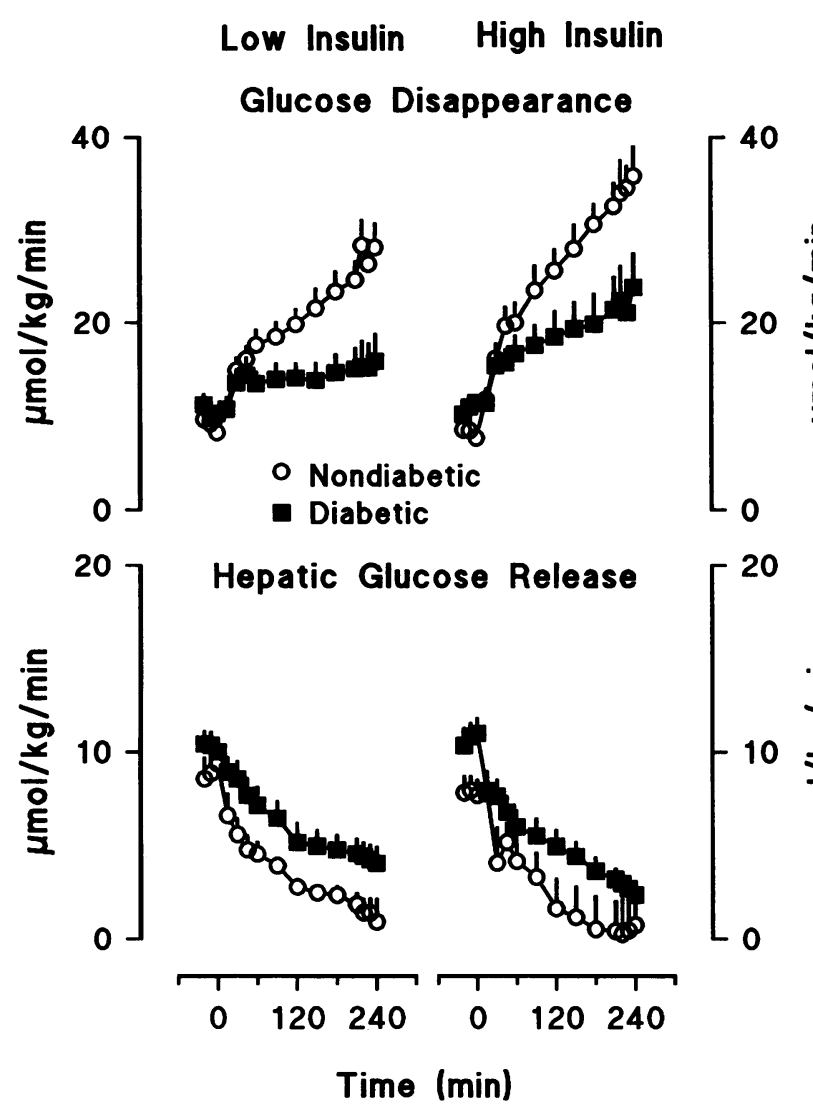

Figure 3. Rates of glucose disappearance and hepatic glucose release observed in the diabetic and nondiabetic subjects during the low (left) and high (right) dose insulin infusions. The low and high dose insulin infusions were started at time zero.

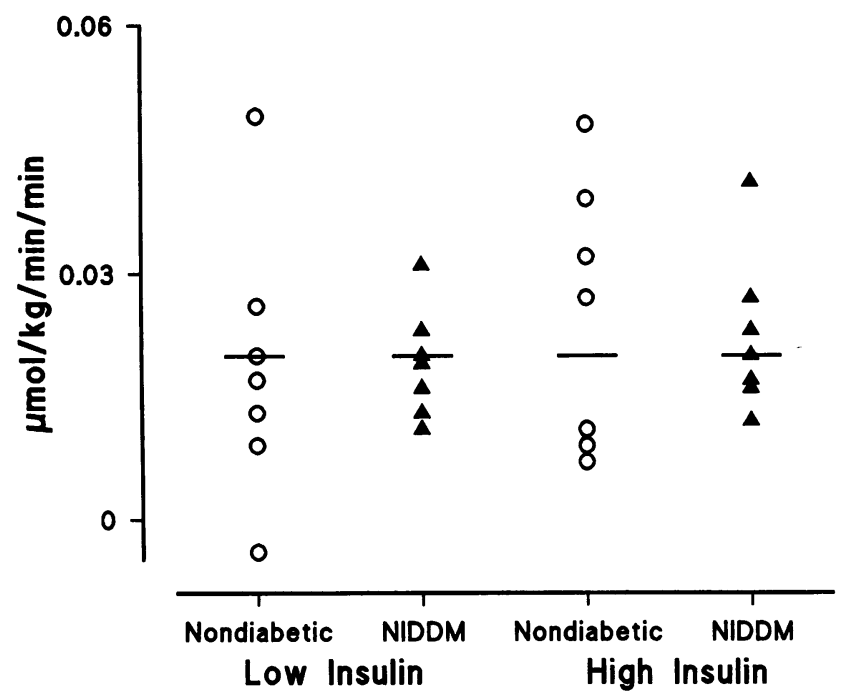

Figure 4. Individual slopes of the rate of suppression of hepatic glucose release versus time observed in the nondiabetic and diabetic subjects during the low and high dose insulin infusions.
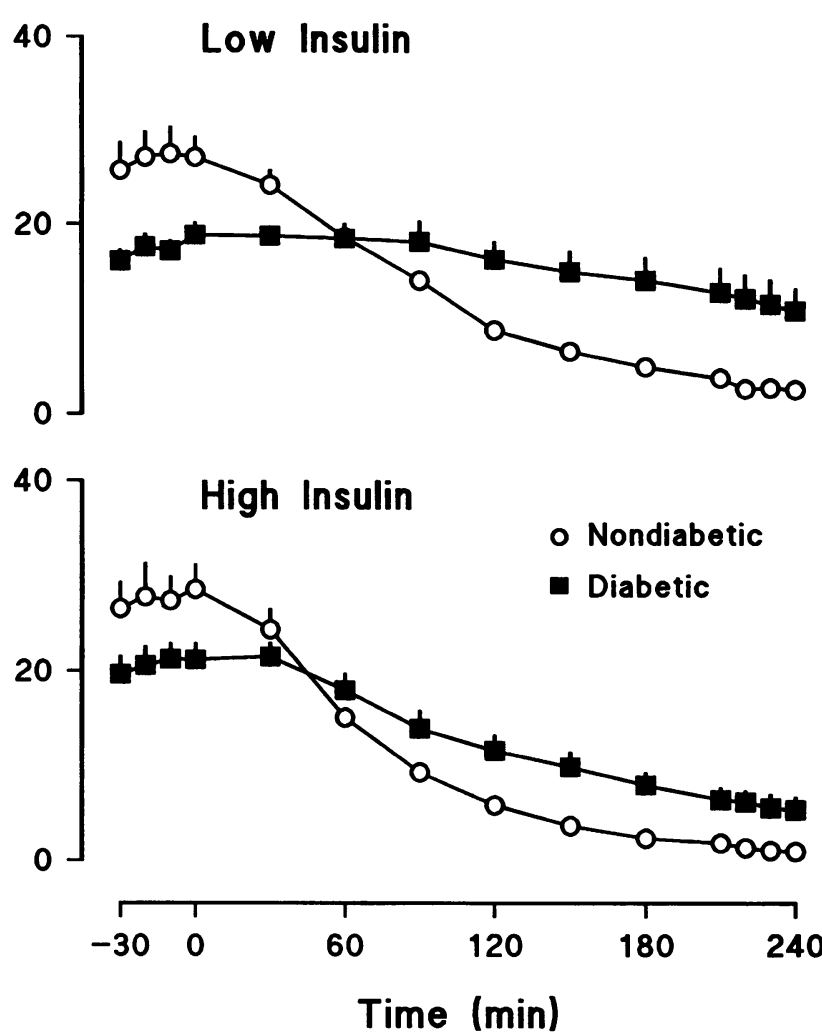

Figure 5. Percent glucose from bicarbonate. The rate of incorporation of ${ }^{14} \mathrm{CO}_{2}$ into systemic glucose observed in the diabetic and nondiabetic subjects during the low (top) and high (bottom) dose insulin infusions. The low and high dose insulin infusions were started at time zero.

virtually no stimulatory effect on glucose disappearance in the diabetic compared to the nondiabetic subjects during the low dose infusion (slope $30-240 \mathrm{~min}$ : $0.01 \pm 0.01$ vs $0.06 \pm 0.01$ $\mu \mathrm{mol} / \mathrm{kg}^{-1}$ per $\min ^{-2}, P<0.001$ ) and a slight but impaired effect during high dose insulin infusion (slope 30-240 min: $0.03 \pm 0.01$ vs $0.09 \pm 0.02 \mu \mathrm{mol} / \mathrm{kg}^{-1}$ per $\min ^{-2} ; P<0.025$ ). This resulted in lower $(P<0.05)$ rates being achieved in diabetic than nondiabetic during the last $30 \mathrm{~min}$ of the insulin infusions.

Percent incorporation of ${ }^{14} \mathrm{CO}_{2}$ into glucose. In an effort to assess the contribution of gluconeogenesis to hepatic glucose release, the rate of incorporation of ${ }^{14} \mathrm{CO}_{2}$ into glucose was measured. The percent glucose derived from ${ }^{14} \mathrm{CO}_{2}$ was lower $(P<0.05)$ in the diabetic than nondiabetic subjects before both the low and high dose insulin infusions. (Fig. 5). Whereas both insulin infusions resulted in a prompt decrease in the percent glucose derived from ${ }^{14} \mathrm{CO}_{2}$ in the nondiabetic subjects, suppression (expressed as area below basal) was impaired $(P<0.01)$ in the diabetic subjects during both the low $(-3.5 \pm 4.3$ vs $-35.9 \pm 5.9 \%)$ and high $(-19.6 \pm 3.4$ vs $-43.7 \pm 6.1 \%)$ insulin infusions. This resulted in a higher $(P<0.01)$ percentage of glucose derived from ${ }^{14} \mathrm{CO}_{2}$ in the diabetic than nondiabetic subjects during the last $30 \mathrm{~min}$ of both the low ( $12 \pm 2$ vs $3 \pm 1 \%$ ) and high $(6 \pm 1$ vs. $1 \pm 0.3 \%)$ dose insulin infusions.

Rate of change of hepatic glucose release and ${ }^{14} \mathrm{CO}_{2}$ incorporation into glucose expressed as a percentage of baseline. In an effort to determine whether suppression of new glucose synthesis occurs in parallel with suppression of hepatic glucose 


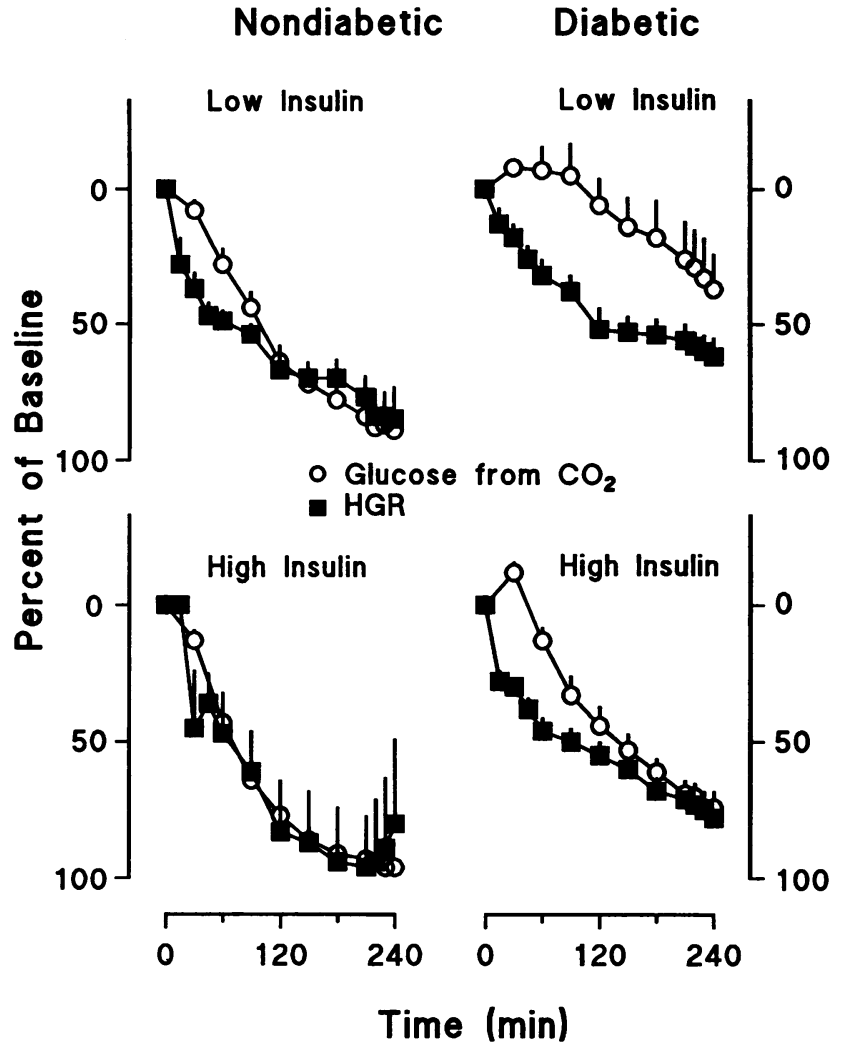

Figure 6. Rates of incorporation of ${ }^{14} \mathrm{CO}_{2}$ (glucose from $\mathrm{CO}_{2}$ ) into glucose and hepatic glucose release expressed as percentage of baseline (mean from -30 to $0 \mathrm{~min}$ ) values in the nondiabetic and diabetic subjects during the low (top) and high (bottom) dose insulin infusions. The low and high dose insulin infusions were started at time zero.

release, both the percent incorporation of ${ }^{14} \mathrm{CO}_{2}$ into glucose and hepatic glucose release were expressed as a percent of basal rates (Fig. 6). When thus expressed, the percent incorporation of ${ }^{14} \mathrm{CO}_{2}$ into glucose in the nondiabetic subjects fell slightly slower than hepatic glucose release during the first $2 \mathrm{~h}$ of the low dose insulin infusion. Suppression of these parameters was comparable during the remainder of the low dose insulin infusion and during the entire high dose insulin infusion. In contrast, the suppression of incorporation of ${ }^{14} \mathrm{CO}_{2}$ into glucose in the diabetic subjects was considerably slower $(P<0.02)$ than suppression of hepatic glucose release throughout the entire low dose insulin infusion. An impaired but less marked discordance between the rate of suppression incorporation of ${ }^{14} \mathrm{CO}_{2}$ into glucose and hepatic glucose release also was observed during the high dose insulin infusion.

Glycerol, lactate, and amino acid concentrations. To determine whether the differences in gluconeogenesis between groups could be due to differences in substrate availability, we measured glycerol, lactate, and amino acid concentrations before and during the low and high dose insulin infusions. The overnight insulin infusion resulted in slightly lower fasting glycerol concentrations in the diabetic than nondiabetic subjects on both the low ( $59 \pm 13$ vs $120 \pm 32 \mathrm{mmol} /$ liter $)$ and high ( $72 \pm 15$ vs $90 \pm 15 \mathrm{mmol} /$ liter) dose insulin study days (Fig. 7). These concentrations; however, did not differ significantly, possibly in part due to large interindividual variation. Fasting lactate,
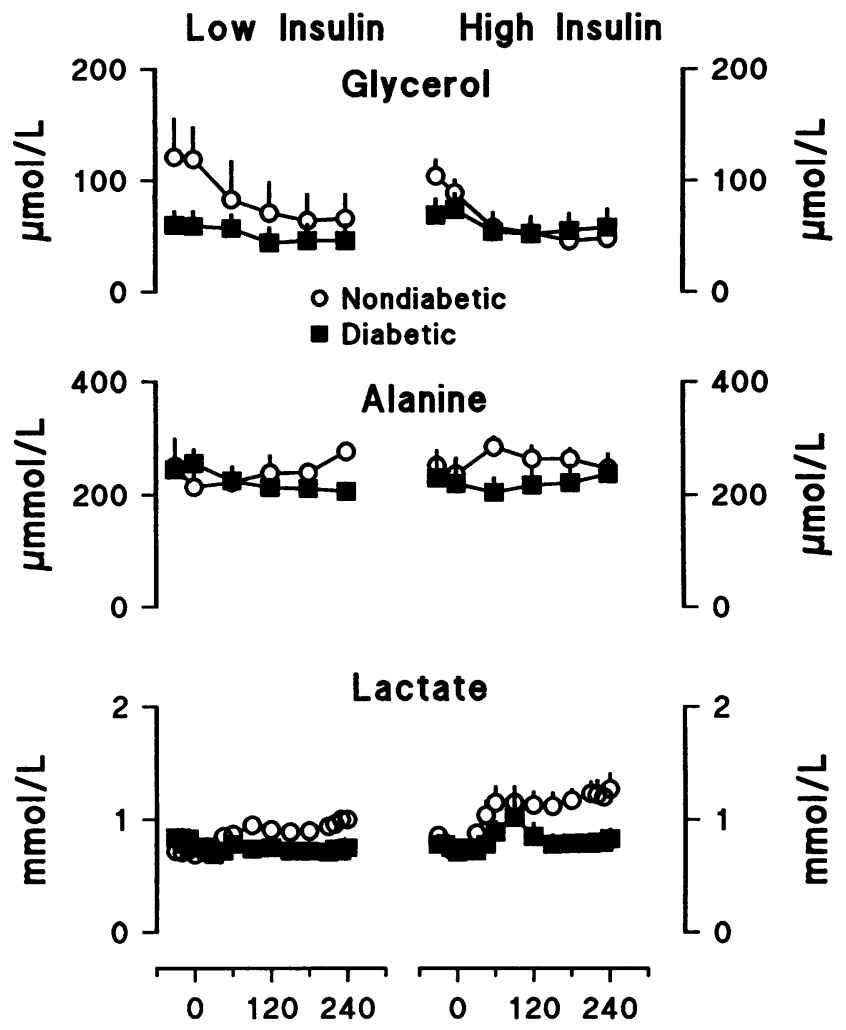

Time (min) Time (min)

Figure 7. Plasma glycerol, alanine, and lactate concentration observed in the diabetic and nondiabetic subjects during the low (left) and high (right) dose insulin infusions. The low and high dose insulin infusions were started at time zero.

alanine, and other amino acid concentrations also did not differ between groups (data not shown).

The insulin infusions resulted in a decrease in glycerol in both groups. Although the magnitude of the fall was less ( $P$ $<0.05$ ) in the diabetic than nondiabetic subjects, the resultant glycerol concentrations did not differ between groups from 60 min onward. Alanine and lactate concentrations if anything were higher in the nondiabetic than diabetic subjects during both insulin infusions (Fig. 7). The concentration of the other amino acids did not differ in diabetic and nondiabetic subjects during both insulin infusions (data not shown).

\section{Discussion}

The present experiments demonstrate that the rate of increase in glucose disappearance in response to an increase in insulin concentration is impaired in people with NIDDM. In contrast, the rate of suppression of hepatic glucose release is normal. Since muscle is the primary site of glucose uptake when insulin is increased in the presence of euglycemia, these data suggest that NIDDM slows the rate-limiting step in insulin action in muscle but not in liver. On the other hand, diabetes impairs the ability of insulin to suppress carbon dioxide incorporation into glucose (a qualitative index of gluconeogenesis) suggesting that while the overall kinetic response to a change in insulin is normal, the relative contribution of gluconeogenesis and glycogenolysis to glucose production is not. 
Effects of diabetes on the rate of stimulation of glucose utilization and suppression of hepatic glucose release. Previous investigators using the hyperinsulinemic euglycemic clamp technique have established that the eventual response achieved at a given insulin concentration is impaired in people with NIDDM (1-5). At steady state, hepatic glucose release is greater and glucose uptake is lower in diabetic than nondiabetic subjects. However, limited information is available regarding the rate of response to insulin in humans due in large part to difficulties in the past in measurement of glucose turnover under non-steady state conditions $(15,28-30)$. The slower rate of increase in glucose disappearance in the diabetic subjects is consistent with results previously reported by DeFronzo et al. using the limb catheterization technique (10). These investigators noted that the rate of increase of leg glucose uptake was slower in diabetic individuals for $1 \mathrm{~h}$ after an acute increase in plasma insulin concentration to $\sim 100 \mu \mathrm{U} / \mathrm{ml}$. The present experiments extend these observations by demonstrating at the whole-body level that this kinetic defect also occurs at lower insulin concentrations and persists for at least $4 \mathrm{~h}$.

In contrast to the slower increase in disposal, NIDDM did not alter the rate of suppression of hepatic glucose release. The rate of fall of glucose release was assessed from $30 \mathrm{~min}$ onward. This period was chosen since, although the insulin concentrations during the infusions did not differ in the two groups, the increment in peripheral and calculated hepatic sinusoidal insulin concentrations during the first $30 \mathrm{~min}$ was slightly lower in the diabetic subjects. This made the comparable rate of fall in the two groups even more impressive. The normal rate of response occurred in the presence of what would traditionally be considered as evidence of hepatic insulin resistance (i.e., higher steady state rates of release of glucose during the final hour of the low dose insulin infusion despite comparable peripheral and calculated hepatic sinusoidal insulin concentrations). A similar dichotomy between the rate of response in hepatic and extrahepatic tissues has also been observed in other insulin resistan states. Prager et al. (31) reported that while the rate of insulininduced stimulation of glucose uptake was impaired in individuals with insulin resistance due to obesity, the rate of suppression of hepatic glucose release was not thus paralleling the pattern observed in the NIDDM subjects in the present experiments.

An overnight insulin infusion was used to lower fasting glucose concentrations in the diabetic subjects to those present in the nondiabetic subjects so that the rate of onset of insulin action could be compared in the presence of comparable and constant glucose concentrations in both groups. This infusion clearly did not restore insulin action to normal since steady state rates of glucose disappearance were lower and hepatic glucose release higher in the diabetic than nondiabetic subjects (see Fig 3 ). However the present experiments do not permit determination of the effects of the overnight insulin infusion on the kinetics of response to insulin. The overnight insulin infusion does not appear to have substantially improved insulin action at steady state since rates of disappearance and hepatic glucose release observed in the present experiments during the final hour of the low dose insulin infusion are virtually identical to those previously observed in a comparable group of diabetic subjects studied under similar conditions in the absence of an overnight insulin infusion (32). However, since the glucose concentrations were permitted to fall in our previous experiments to the euglycemic range during the insulin infusion (32), the kinetics of response to insulin could not be assessed. It is therefore possible that the overnight insulin infusion altered the kinetic but not the eventual response to insulin. Although future studies will be required to answer this question, the present data clearly demonstrate that insulin resistance can have different effects on the kinetics of insulin action in hepatic and extrahepatic tissues. Despite impaired stimulation of glucose utilization, the rate of suppression of hepatic glucose release in response to a change in insulin concentration in people with NIDDM is still normal.

Multiple steps are required for insulin to exert it actions. Insulin must transverse a capillary epithelial barrier in muscle but not liver (33-38). Although the rate of transcapillary transport of insulin has not been measured in diabetic humans, slowed egress of insulin from blood into the interstitium is unlikely to be the explanation for the impaired extrahepatic responses observed in the present experiments since it has not been impaired in other insulin resistant states in which it has been measured $(39,40)$. Furthermore, it seems unlikely that a mere delay in equilibration could fully explain the present data since the difference between responses in the diabetic and nondiabetic subjects presumably would decrease with time rather than progressively diverge as was observed. This pattern suggests additional defects at the level of insulin binding and/or intracellular signaling. Such defects may be intrinsic to NIDDM or may be influenced by factors associated with the diabetic state (e.g., the severity of hyperglycemia and/or insulin deficiency).

Insulin is believed to stimulate glucose uptake in muscle by increasing either the number or activity of plasma membrane glucose transporters (41). In contrast, insulin decreases hepatic glucose release by decreasing the activity of glycogenolytic and/or gluconeogenetic enzymes. The slower rate of stimulation of glucose disposal but normal rate of suppression of glucose release could readily be explained by an alteration in the rate of activation or in the rate of incorporation of the insulin sensitive glut 4 isomer into the plasma membrane since this transporters is believed to regulate glucose metabolism in muscle but not liver (41). Against this supposition is the demonstration by Ciraraldi et al. that the kinetics of insulin stimulated adipocyte 3-O-methylglucose uptake did not differ in comparably obese diabetic and nondiabetic subjects (42). Whatever the cause, the dichotomy between the slowed rate of uptake but the normal rate of suppression of glucose release is of interest relative to the recently proposed single-gateway hypothesis in which it is postulated that peripheral insulin action is the primary determinant of hepatic response (43). The discordance in the diabetic subjects between the normal rate of suppression of hepatic glucose release and the abnormal rate of increase in glucose uptake indicates that the close linkage between hepatic and extrahepatic responses observed in nondiabetic humans is disrupted by NIDDM.

Effect of insulin on the contribution of gluconeogenesis to hepatic glucose release in diabetes. Both glycogenolysis and gluconeogenesis contribute to hepatic glucose release after an overnight fast. Gluconeogenesis appears to be increased in untreated diabetes mellitus $(11,12)$. Yki-Järvinen et al. have reported that treatment of people with NIDDM with subcutaneous insulin decreases the proportion of lactate converted to glucose (44). The current studies extend this observation by demonstrating that an overnight infusion of insulin in amounts sufficient to maintain euglycemia can lower the rate of incorporation of ${ }^{14} \mathrm{CO}_{2}$ into glucose to that observed in nondiabetic individuals fasted for a comparable period of time. As discussed in detail 
elsewhere $(25,26)$, incorporation of radiolabeled carbon dioxide into the carbon skeleton of glucose occurs as pyruvate is converted to oxaloacetate via pyruvate carboxylase. Similar to other such approaches, this method provides a qualitative rather than a quantitative estimate of gluconeogenesis. Although the percentage of glucose derived from ${ }^{14} \mathrm{CO}_{2}$ was slightly lower in the diabetic subjects during the basal period, the rate of hepatic glucose release was slightly higher; therefore the absolute rate of release of $\left[{ }^{14} \mathrm{C}\right]$ glucose did not differ between groups. However the response during the insulin infusions was clearly abnormal in the diabetic subjects. Whereas both the low and high insulin infusions resulted in a prompt decrease in the rate of incorporation of ${ }^{14} \mathrm{CO}_{2}$ into glucose in the nondiabetic subjects, virtually no suppression occurred in the diabetic subjects during the low dose insulin infusion and minimal but still impaired suppression occurred during the higher insulin infusion. The disproportionate contribution of gluconeogenesis to glucose release is unlikely to be substrate driven, as the concentrations of glycerol, lactate, alanine, and other glycogenic amino acids were not increased in the diabetic subjects. In addition, it does not appear to be hormonally mediated, since suppression of glucagon was equivalent in both groups and since cortisol and growth hormone concentrations did not change during insulin infusion in either group. These data strongly suggest that the site of the defective regulation of gluconeogenesis by insulin is intrahepatic in origin.

Several recent studies have reported that glycogenolysis and gluconeogenesis are regulated in a reciprocal manner under a variety of circumstances (45-48). Although the rate of incorporation of ${ }^{14} \mathrm{CO}_{2}$ into glucose was excessive in diabetic subjects during both insulin infusions, as discussed above, the rate of suppression of hepatic glucose release by insulin was normal. When the rates of incorporation of ${ }^{14} \mathrm{CO}_{2}$ into glucose and hepatic glucose release were expressed as a percentage of baseline (Fig. 6), both fell in parallel in the nondiabetic subjects, implying proportional suppression of glycogenolysis and gluconeogenesis. In contrast, glucose derived from carbon dioxide decreased at a slower rate than hepatic glucose release in the diabetic subjects suggesting that the delay in suppression of gluconeogenesis was offset by more rapid suppression of glycogenolysis. The data expressed in this fashion suggest that glycogenolysis was particularly sensitive to further increases in insulin in these well-insulinized patients with NIDDM. This conclusion, however, must remain tentative until the effects of insulin on glycogenolysis and gluconeogenesis are measured quantitatively rather than qualitatively.

Limitations. It is possible that the rate of suppression may have been impaired in the diabetic subjects if assessed during lower insulin concentrations. However, the low insulin infusion rate used in the current studies resulted in insulin concentrations that were only $\sim 15 \mu \mathrm{U} / \mathrm{ml}$ higher than those required to maintain euglycemia in the basal state. $\left[6-{ }^{3} \mathrm{H}\right]$ glucose specific activity increased slightly in both groups during the insulin infusions. Previous studies have shown that an increase of this degree has a minimal impact on the accuracy of measurement of glucose turnover $(15,28)$. Even if it did, the slightly greater increase in glucose specific activity in the diabetic group would lead to an overestimate of the rate of increase in disappearance and an underestimate of the rate of decrease in glucose release; such errors would strengthen the present conclusions.

Quantitative measurement of gluconeogenesis in humans is difficult if not impossible. The advantages and limitations of using the rate of incorporation of labeled carbon dioxide into glucose as an index of gluconeogenesis have been previously discussed $(16,25,26)$. The present experiments only provide an estimate of the proportion of glucose entering the systemic circulation that is derived from gluconeogenesis. Chiasson et al. have previously shown that small increments in insulin decrease the rate of release of glucose derived from alanine in humans fasted for $48 \mathrm{~h}$ without fully suppressing incorporation of labeled glucose into glycogen (49). Thus we cannot exclude the possibility that insulin preferentially shunted glucose-6phosphate synthesized via the gluconeogenic pathway into glycogen in the nondiabetic but not the diabetic subjects.

In summary, hepatic glucose release is inappropriately high and glucose disappearance is inappropriately low for the prevailing insulin level in individuals with NIDDM, indicating the presence of insulin resistance. The rate of insulin-induced stimulation of glucose disappearance is slower in diabetic than nondiabetic subjects, whereas the rate of suppression of hepatic glucose release is normal. On the other hand, diabetes impairs the ability of insulin to decrease the rate of entry of newly synthesized glucose into the systemic circulation, thereby changing the relative contribution of gluconeogenesis and glycogenolysis to hepatic glucose release. These data indicate that in people with NIDDM, the rate-limiting steps in insulin action and therefore the potential sites of insulin resistance differ in hepatic and extrahepatic tissues.

\section{Acknowledgments}

We thank E. Butkiewicz, J. King, T. Madson, and D. Nash for their technical assistance, A. Pelot for her assistance in preparation of the manuscript, and the staff of the Mayo Clinical Research Center for their assistance in performing the studies. This work was supported by U.H.P.S. (grants DK29953, DK41973, RR00585) and the Mayo Foundation. Dr. Aus A. Alzaid was supported by a mentor-based American Diabetes Association Research Fellowship award and by the Armed Forces Hospital, Riyadh, Saudi Arabia.

\section{References}

1. Bogardus, C., S. Lillioja, B. Howard, G. Reaven, and D. Mott. 1984 Relationships between insulin secretion, insulin action, and fasting plasma glucose concentration in nondiabetic and non-insulin-dependent diabetic subjects. J. Clin. Invest. 74:1238-1246.

2. Kolterman, O., R. Gray, J. Griffin, P. Burstein, J. Insel, J. Scarlett, and J. Olefsky. 1981. Receptor and postreceptor defects contribute to the insulin resistance in non-insulin dependent diabetes mellitus. J. Clin. Invest. 68:957-965.

3. Groop, L., R. Bonndonna, S. Delprato, K. Ratheiser, K. Zyck, E. Ferrannini, and R. DeFronzo. 1989. Glucose and free fatty acid metabolism in non-insulindependent diabetes mellitus: evidence for multiple sites of insulin resistance. $J$. Clin. Invest. 84:205-213.

4. Firth, R., P. Bell, and R. Rizza. 1987. Insulin action in non-insulin-dependent diabetes mellitus: the relationship between hepatic and extrahepatic insulin resistance and obesity. Metab. Clin. Exp. 36:1091-1095.

5. Campbell, P., L. Mandarino, and J. Gerich. 1988. Quantification of the relative impairment of actions of insulin on hepatic glucose production and peripheral glucose uptake in non-insulin-dependent diabetes mellitus. Metab. Clin. Exp. 37:15-21.

6. Radziuk, J., M. J. McDonald, D. Rubenstein, and J. Dupre. 1978. Initia splanchnic extraction of ingested glucose in normal man. Metab. Clin. Exp. 27:657-669.

7. Firth, R., P. Bell, H. Marsh, I. Hansen, and R. Rizza. 1986. Postprandia hyperglycemia in patients with non-insulin-dependent diabetes mellitus. J. Clin. Invest. 77:1525-1532.

8. Ferrannini, E., D. Simonson, L. Katz, G. Reichard, S. Bevilacqua, E. Barrett, M. Olsson, and R. DeFronzo. 1988. The disposal of an oral glucose load in patients with non-insulin-dependent diabetes. Metab. Clin. Exp. 37:79-85.

9. Rizza, R. A., L. J. Mandarino, and J. E. Gerich. 1981. Dose-response 
characteristics for effects of insulin on production and utilization of glucose in man. Am. J. Physiol. 240:E630-E639.

10. Calles-Escandon, J., and D. C. Robbins. 1987. Loss of early phase of insulin release in humans impairs glucose tolerance and blunts thermic effects of glucose. Diabetes. 36:1167-1172.

11. DeFronzo, R. A., R. Gunnarsson, O. Björkman, M. Olsson, and J. Wahren 1985. Effects of insulin on peripheral and splanchnic glucose metabolism in noninsulin-dependent (type II) diabetes mellitus. J. Clin. Invest. 76:149-155.

12. Consoli, A., N. Nurjhan, F. Capani, and J. Gerich. 1989. Predominant role of gluconeogenesis in increased hepatic glucose production in NIDDM. Diabetes. 38:550-557.

13. Magnusson, I., D. L. Rothman, L. D. Katz, R. G. Shulman, and G. I Shulman. 1992. Increased rate of gluconeogenesis in type II diabetes mellitus. $J$. Clin. Invest. 90:1323-1327.

14. White, N., D. Skor, and J. Santiago. 1982. Practical closed-loop insulin delivery. A system for the maintenance of overnight euglycemia and the calculation of basal insulin requirements insulin-dependent diabetes. Ann. Intern. Med. 97:210-213.

15. Butler, P., A. Caumo, A. Zerman, P. O'Brien, C. Cobelli, and R. A. Rizza 1993. Methods for assessment of the rate of onset and offset of insulin action during non-steady state in man. Am. J. Physiol. 264:E548-E560.

16. McMahon, M., H. M. Marsh, and R. A. Rizza. 1989. Effects of basa insulin supplementation on disposition of mixed meal in obese patients with NIDDM. Diabetes. 38:291-303.

17. Butler, P., E. Kryshak, and R. Rizza. 1991. Mechanism of growth hormoneinduced postprandial carbohydrate intolerance in humans. Am. J. Physiol. 260:E513-E520.

18. McGowen, M., J. Artiss, D. Strandberg, and B. Zak, 1983. A peroxidasecoupled method for the colorimetric determination of serum triglycerides. Clin. Chem. 29:538-542.

19. Jones, B., and J. Gilligan. 1983. Amino acid analysis by o-pthaldehyde precolumn derivitization and reversed phase HPLC. Am. Biotechnol. Lab. 12:4551.

20. Adkins-Marshall, B., M. J. Pagliasotti, J. R. Asher, C. C. Connolly D. W. Neal, P. E. Meyers, G. K. Hendrik, R. B. Adkins, Jr., and A. D. Cherrington 1992. Role of hepatic nerves in response of liver to intraportal glucose delivery in dogs. Am. J. Physiol. 262 (Endocrinol. Metab. 25):E679-E686.

21. Hother-Nielsen, O. Schmitz, J. Bak, and H. Beck-Nielsen. 1987. Enhanced hepatic sensitivity, but peripheral insulin resistance in patients with type 1 (insulin-dependent) diabetes. Diabetologia. 30:834-840.

22. De Bodo, R. C., R. Steele, N. Altszuler, A. Dunn, and J. S. Bishop. 1963 On the hormonal regulation of carbohydrate metabolism: studies with $\mathrm{C}^{14}$ glucose Recent Prog. Horm. Res. 19:445-488.

23. Steele, R., J. Wall, R. De Bodo, and N. Altszuler. 1956. Measurement of size and turnover rate of body glucose pool by the isotope diluation method. Am. J. Physiol. 187:15-24.

24. Bradley, D. C., G. M. Steil, and R. N. Bergman. 1993. Quantification of measurement error with optimal segments: basis for adaptive time course smoothing. Am. J. Physiol. 264:E902-911.

25. Brosnan, J. T. 1982. Pathways of carbon flux in gluconeogenesis. Fed. Proc. 41:91-95.

26. Katz, J. 1985. Determination of gluconeogenesis in vivo with ${ }^{14} \mathrm{C}$-labeled substrates. Am. J. Physiol. 248:R391-R399.

27. Puhakainen, I. V. A. Koivisto, and H. Yki-Järvinen. 1992. Lipolysi and gluconeogenesis from glycerol are increased in patients with non-insulindependent diabetes mellitus. J. Clin. Endocrinol. Metab. 75:789-794.

28. Finegood, D., R. Bergman, and M. Vranic. 1987. Estimation of endogenous glucose production during hyperinsulinemic-euglycemic glucose clamps: comparison of unlabelled and labelled exogenous glucose infusates. Diabetes. 36:914924.
29. Bergman, R., D. Finegood, and M. Ader. 1985. Assessment of insulin sensitivity in vivo. Endocr. Rev. 6:45-86.

30. Katz, H., P. Butler, M. Homan, A. Zerman, A. Caumo, C. Cobelli, and R. Rizza. 1993. Hepatic and extrahepatic insulin action in humans: measurement in the absence of non-steady-state error. Am. J. Physiol. 264:E561-E566.

31. Prager, P., P. Wallace, and J. M. Olefsky. 1986. In vivo kinetics of insulin action on peripheral glucose disposal and hepatic glucose output in normal and obese subjects. J. Clin. Invest. 78:472-481.

32. Butler, P., E. J. Kryshak, W. F. Schwenk, M. W. Haymond, and R. A Rizza. 1990. Hepatic and extrahepatic responses to insulin in NIDDM and nondiabetic humans: assessment in the absence of artifact introduced by tritiated nonglucose contaminants. Diabetes. 39:217-225.

33. Chernick, S. S., R. J. Gardiner, and R. O. Scow. 1987. Restricted passage of insulin across capillary endothelium in perfused rat adipose tissue. Am. J. Physiol. 253:E475-E480.

34. Yang, Y., I. Hope, M. Ader, and R. Bergman. 1989. Insulin transport across capillaries is rate limiting for insulin action in dogs. J. Clin. Invest. 84:1620-1628.

35. Bliar, H. 1949. A re-examination of the structure of mammalian liver. Am. J. Anat. 85:379-456.

36. Jansson, P-A. E., J. P. Fowelin, H. P. Von Schenck, U. P. Smith, and P. N. Lönnroth. 1993. Measurement by microdialysis of the insulin concentration in subcutaneous interstitial fluid. Diabetes. 42:1469-1473.

37. Freidenberg, G. R., S. Suter, R. R. Henry, J. Nolan, D. Reichart, and J. M. Olefsky. 1994. Delayed onset of insulin activation of the insulin receptor kinase in vivo in human skeletal muscle. Diabetes. 43:118-126.

38. Bar, R. S., J. C. Hoak, and M. L. Peacock. 1978. Insulin receptors in human endothelial cells: identification and characterization. J. Clin. Endocrinol. 47:699-702.

39. Bryer-Ash, M. 1993. Altered distribution of insulin between intravascular and interstitial compartments in STZ-induced diabetic rats. Diabetes. 42:1528 1535.

40. Castillo, C., C. Bogardus, R. Bergman, P. Thuillez, and S. Lillioja. 1994 Interstitial insulin concentrations determine glucose uptake rates but not insulin resistance in lean and obese men. J. Clin. Invest. 93:10-16.

41. Kahn, B. 1992. Facilitative glucose transporters: regulatory mechanisms and dysregulation in diabetes. J. Clin. Invest. 89:1367-1374.

42. Ciaraldi, T. P., J. M. Molina, and J. M. Olefsky. 1991. Insulin action kinetics in adipocytes from obese and non-insulin-dependent diabetes mellitus subjects: identification of multiple cellular defects in glucose transport. J. Clin Endocrinol. \& Metab. 72:876-882.

43. Poulin, R. A., G. M. Steil, D. M. Moore, M. Ader, and R. N. Bergman 1994. Dynamics of glucose production and uptake are more closely related to insulin in hindlimb lymph than in thoracic duct lymph. Diabetes. 43:180-190.

44. Yki-Järvinen, H., E. Helve, T. Sane, N. Nurjhan, and M-R. Taskinen. 1989. Insulin inhibition of overnight glucose production and gluconeogenesis from lactate in NIDDM. Am. J. Physiol. 256:E732-E739.

45. Jenssen, T., N. Nurjahan, A. Consoli, and J. E. Gerich. 1990. Failure of substrate-induced gluconeogenesis to increase overall glucose appearance in normal humans. J. Clin. Invest. 86:489-497.

46. Puhakainen, I., and H. Yki-Järvinen. 1993. Inhibition of lipolysis decreases lipid oxidation and gluconeogenesis from lactate but not fasting hyperglycemia or total hepatic glucose production in NIDDM. Diabetes. 42:1694-1699.

47. Clore, J. N., P. S. Glickman, J. E. Nestler, and W. G. Blackard. 1991. In vivo evidence for hepatic autoregulation during FFA-stimulated gluconeogenesis in normal humans. Am. J. Physiol. 261:E425-E429.

48. Clore, J. N., and W. G. Blackard. 1994. Suppression of gluconeogenesis after a 3-day fast does not deplete liver glycogen in patients with NIDDM. Diabetes. 43:256-262.

49. Chiasson, J. L., R. L. Atkinson, A. D. Cherrington, U. Keller, B. C Sinclair-Smith, W. W. Lacy, and J. E. Liljenquist. 1980. Effects of insulin at two dose levels on gluconeogenesis from alanine in fasting man. Metab. Clin. Exp. 29:810-818. 